\title{
Correction: Loss of EGFR signaling-regulated miR-203 promotes prostate cancer bone metastasis and tyrosine kinase inhibitors resistance
}

\author{
Man Kit Siu ${ }^{1,2, *}$, Wassim Abou-Kheir ${ }^{3, *}$, Juan Juan Yin ${ }^{4, *}$, Yung-Sheng Chang ${ }^{1}$, Ben \\ Barrett $^{4}$, Florent Suau ${ }^{4}$, Orla Casey ${ }^{4}$, Wei-Yu Chen ${ }^{5}$, Lei Fang ${ }^{4}$, Paul Hynes ${ }^{4}$, Yao- \\ Yu Hsieh ${ }^{1,6,9}$,Yen-Nien Liu ${ }^{1,7}$, Jiaoti Huang ${ }^{8}$ and Kathleen Kelly ${ }^{4}$ \\ ${ }^{1}$ Graduate Institute of Cancer Biology and Drug Discovery, College of Medical Science and Technology, Taipei Medical \\ University, Taipei, Taiwan \\ 2 Department of Anesthesiology, Wan Fang Hospital, Taipei Medical University, Taipei, Taiwan \\ ${ }^{3}$ Department of Anatomy, Cell Biology and Physiological Sciences Faculty of Medicine, American University of Beirut, Beirut, \\ Lebanon \\ ${ }^{4}$ Cell and Cancer Biology Branch, National Cancer Institute, National Institutes of Health, Bethesda, MD, USA \\ ${ }^{5}$ Department of Pathology, Wan Fang Hospital, College of Medicine, Taipei Medical University, Taipei, Taiwan \\ ${ }^{6}$ Division of Hematology and Oncology, Shuang Ho Hospital, Taipei Medical University, New Taipei City, Taiwan \\ ${ }^{7}$ Center of Excellence for Cancer Research, Wan Fang Hospital, College of Medicine, Taipei Medical University, Taipei, Taiwan \\ 8 Department of Pathology and Laboratory Medicine, University of California, Los Angeles, CA, USA \\ ${ }^{9}$ Department of Internal Medicine, Division of Hematology and Oncology, School of Medicine, College of Medicine, Taipei \\ Medical University, Taipei, Taiwan \\ * These authors contributed equally to this work \\ Published: August 17, 2018
}

Copyright: Siu et al. This is an open-access article distributed under the terms of the Creative Commons Attribution License 3.0 (CC BY 3.0), which permits unrestricted use, distribution, and reproduction in any medium, provided the original author and source are credited.

This article has been corrected: The author affiliation is given below:

\section{Yao-Yu Hsieh ${ }^{1,6,9}$}

${ }^{9}$ Department of Internal Medicine, Division of Hematology and Oncology, School of Medicine, College of Medicine, Taipei Medical University, Taipei, Taiwan

Original article: Oncotarget. 2014; 5:3770-3784. https://doi.org/10.18632/oncotarget.1994 